IJMMS 25:11 (2001) 753-756

PII. S0161171201003969

http://ijmms.hindawi.com

(c) Hindawi Publishing Corp.

\title{
MULTIMODAL CYCLES WITH LINEAR MAP HAVING EXACTLY ONE FIXED POINT
}

\author{
IRENE MULVEY
}

(Received 5 October 1999)

\begin{abstract}
We describe a class of cycles that cannot be forced by a cycle whose linear map has exactly one fixed point.
\end{abstract}

2000 Mathematics Subject Classification. Primary 37E05, 37E15.

1. Introduction. This note is concerned with the forcing relation on cycles. In particular, we consider cycles $\theta$ for which the $\theta$-linear map has exactly one fixed point. We prove a theorem which describes a large class of cycles that cannot be forced by $\theta$.

2. Definitions. Throughout this note, $f: I \rightarrow I$ denotes a continuous map of a compact interval. For $x \in I, f^{0}(x)=x$, and for $n \in N, f^{n}(x)=f\left(f^{n-1}(x)\right)$. An element $x \in I$ is a periodic point for $f$ if there exists $k \in N$ satisfying $f^{k}(x)=x$. The least such $k$ is called the period of $x$. A point of period 1 is called a fixed point. The orbit of $x \in I$ is the set $\left\{f^{n}(x)\right\}_{n=0}^{\infty}$ and is denoted $O(x)$. If $x$ is periodic with period $k$, then $\mathcal{O}(x)$ is a finite set consisting of $k$ distinct elements.

A cycle of order $n$ is a bijection $\theta:\{1,2, \ldots, n\} \rightarrow\{1,2, \ldots, n\}$ satisfying $\theta^{k}(1) \neq$ 1 for $1 \leq k<n$. Let $x$ be a periodic point for $f$ with least period $n$ and $\mathcal{O}(x)=$ $\left\{x_{1}<x_{2}<\cdots<x_{n}\right\}$. We say that $x$ has orbit type $\theta$ if $\theta$ is a cycle of order $n$ and $f\left(x_{i}\right)=x_{\theta(i)}$ for $1 \leq i \leq n$. In this case, we also say that the periodic orbit $\mathcal{O}(x)$ has orbit type $\theta$. We say that $f$ has a periodic orbit of orbit type $\theta$ if there exists a periodic point $x \in I$ which has orbit type $\theta$. A cycle $\theta$ forces a cycle $\eta$ if whenever $f$ has a periodic orbit of type $\theta, f$ has a periodic point of type $\eta$.

For a cycle $\theta$ of order $n$, the $\theta$-linear map $L_{\theta}:[1, n] \rightarrow[1, n]$ is defined by

$$
\begin{gathered}
L_{\theta}(k)=\theta(k), \quad \text { for } 1 \leq k \leq n, \\
L_{\theta} \text { is linear on }[i, i+1], \text { for } 1 \leq i \leq n-1 .
\end{gathered}
$$

The graph of $L_{\theta}$ consists of at most $n-1$ linear segments, each having a slope $m$ satisfying $|m| \geq 1$. A cycle $\eta$ is forced by $\theta$ if and only if $L_{\theta}$ has a periodic orbit of type $\eta[1]$.

Baldwin [2] defined the forcing relation and proved that the forcing relation induces a partial order on the set of cycles. He provided an exhaustive but inefficient algorithm for determining whether one cycle forces another. Jungreis [6] provided a combinatorial method to determine if one cycle forces another in certain cases. In [3] a geometric version of Jungreis's algorithm is given and in [4] this algorithm is generalized to any 
two cycles. In [8], another geometric algorithm is given to determine the forcing relation. This algorithm is similar to Baldwin's original algorithm but more efficient. A cycle is called unimodal if $L_{\theta}$ has exactly one turning point (a maximum, say). In [5] the forcing relation on the set of unimodal cycles is studied. In particular, it is shown that the forcing relation induces a total order on the set of unimodal cycles. In $[7,9]$ the structure of this totally ordered set is investigated.

3. Preliminaries. In this section, we define the $R L$-pattern for any cycle, and we define the step number for a cycle $\theta$ for which $L_{\theta}$ has exactly one fixed point.

DEFINITION 3.1. Let $\eta$ be any cycle of order $k$. The $R L$-pattern for $\eta$ is the sequence

$$
G=G_{1} G_{2} \cdots G_{k} \in\{R, L\}^{k}
$$

defined by

$$
G_{i}= \begin{cases}R & \text { if } \eta^{i}(1)>\eta^{i-1}(1), \\ L & \text { if } \eta^{i}(1)<\eta^{i-1}(1) .\end{cases}
$$

Let $R(\eta)$ denote the length of the longest string of consecutive $R$ 's in the $R L$-pattern for $\eta$.

Obviously, every $R L$-pattern begins with an $R$ and ends with an $L$.

Let $\theta$ be a cycle of order $n$ such that $L_{\theta}$ has exactly one fixed point. Let $p_{1} \in(1, n)$ denote the unique fixed point and let $E_{1}=\left\{x<p_{1} \mid f(x)=p_{1}\right\}$. If $E_{1} \neq \varnothing$, we let $p_{2}=\max \left\{E_{1}\right\}$. For $i>1$, if the points $p_{1}, p_{2}, \ldots, p_{i}$ and nonempty sets $E_{1}, \ldots, E_{i-1}$ have been defined, we set

$$
E_{i}=\left\{x<p_{i} \mid f(x)=p_{i}\right\}
$$

If $E_{i} \neq \varnothing$, we let $p_{i+1}=\max \left\{E_{i}\right\}$. We see that for some $i \geq 1, E_{i}=\varnothing$, for otherwise, there would exist a strictly decreasing sequence $\left\{p_{n}\right\}_{n=1}^{\infty}$ in $[1, n]$, converging to a point $p<p_{1}$ but satisfying, for each $n$,

$$
L_{\theta}\left(p_{n}\right)=p_{n-1}
$$

so that by continuity,

$$
\lim _{n \rightarrow \infty} L\left(p_{n}\right)=L(p)
$$

and at the same time

$$
\lim _{n \rightarrow \infty} L\left(p_{n}\right)=\lim _{n \rightarrow \infty} p_{n-1}=p .
$$

Thus $L(p)=p$, which would contradict the assumption that $L_{\theta}$ has exactly one fixed point. Therefore we can make the following definition.

DEFINITION 3.2. Let $\theta$ be a cycle of order $n$ such that $L_{\theta}$ has exactly one fixed point. The step number of $\theta$, denoted $S(\theta)$, is the (smallest) value of $i$ for which $E_{i}=\varnothing$.

EXAMPLE 3.3. The cycle $\eta_{1}=\left(\begin{array}{llll}1 & 2 & 3 & 4\end{array}\right)$ has $R L$-pattern $R R R L$. The cycle $\eta_{2}=$ (1 472685 ) has $R L$-pattern RRLRLRLL; $R\left(\eta_{1}\right)=3$ and $R\left(\eta_{2}\right)=2$. 
4. Results. For any cycle $\theta$ such that $L_{\theta}$ has exactly one fixed point, the following theorem describes a large class of cycles that cannot be forced by $\theta$.

THEOREM 4.1. Let $\theta$ be a cycle of order $n \geq 2$ such that $L_{\theta}$ has exactly one fixed point. Let $S(\theta)$ denote the step number of $\theta$. Let $\eta$ be any cycle. If $R(\eta)>S(\theta)$, then $\theta$ does not force $\eta$.

Proof. We have

$$
1<p_{S(\theta)}<p_{S(\theta)-1}<\cdots<p_{2}<p_{1}<n
$$

We write

$$
[1, n]=\bigcup_{i=1}^{S(\theta)+1} I_{i}
$$

where

$$
\begin{gathered}
I_{1}=\left[p_{1}, n\right], \\
I_{i}=\left[p_{i}, p_{i-1}\right] \quad \text { for } 2 \leq i \leq S(\theta), \\
I_{S(\theta)+1}=\left[1, p_{S(\theta)}\right] .
\end{gathered}
$$

For any $x \in \operatorname{int}\left(I_{1}\right), L_{\theta}(x)<x$. So $x$ cannot be the leftmost point in any periodic orbit. For $2 \leq i \leq S(\theta)+1$, we argue inductively. If $x \in \operatorname{int}\left(I_{i}\right)$, then $L_{\theta}(x)>x$ and $L_{\theta}(x) \in$ $\bigcup_{j=1}^{i-1} I_{j}$, so if $x$ is the leftmost point of a periodic orbit of type $\gamma$, the $R L$-pattern of $\gamma$ consist of at most $i-1$ consecutive $R$ 's followed by an $L$. That is, $R(\gamma) \leq i-1$. This shows that any cycle $\eta$ forced by $\theta$ must have $R(\eta) \leq S(\theta)$.

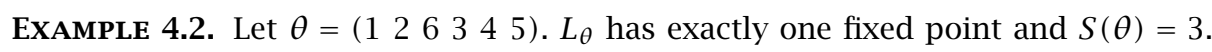
From Theorem 4.1, we know that for all $n \geq 5, \theta$ does not force $\left(\begin{array}{llll}1 & 2 & 3 & \cdots\end{array}\right.$ ). Using the technique developed in [8] it is seen that $\theta$ does force $\left(\begin{array}{lll}1 & 2 & 3\end{array} 4\right)$ and that there are

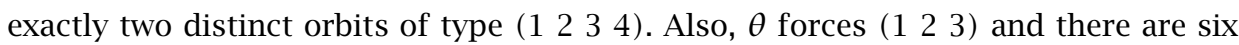

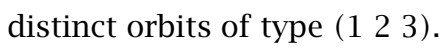

EXAMPLE 4.3. Let $\theta=\left(\begin{array}{ll}1 & 3528476\end{array}\right)$. $L_{\theta}$ has one fixed point and $S(\theta)=2$. From Theorem 4.1, we see that for all $n \geq 4, \theta$ does not force ( $\begin{array}{llll}1 & 2 & 3 & \cdots\end{array}$ ). Using [8], one can find exactly two distinct orbits of type (l $\left.\begin{array}{llll}1 & 2 & 4 & 3\end{array}\right)$, exactly fourteen distinct orbits of type (1 3224 ), exactly eleven distinct orbits of type (1 $\left.\begin{array}{llll}1 & 2 & 3\end{array}\right)$ and one can show that

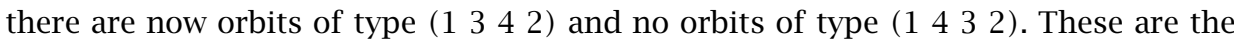
only orbit types of period 4 forced by $\theta$.

\section{REFERENCES}

[1] L. Alsedà, J. Llibre, and M. Misiurewicz, Combinatorial Dynamics and Entropy in Dimension One, Advanced Series in Nonlinear Dynamics, vol. 5, World Scientific Publishing, New Jersey, 1993. MR 95j:58042. Zbl 843.58034.

[2] S. Baldwin, Generalizations of a theorem of Šarkovskiı̆ on orbits of continuous real-valued functions, Discrete Math. 67 (1987), no. 2, 111-127. MR 89c:58057. Zbl 632.06005.

[3] C. Bernhardt, E. Coven, M. Misiurewicz, and I. Mulvey, Comparing periodic orbits of maps of the interval, Trans. Amer. Math. Soc. 333 (1992), no. 2, 701-707. MR 92m:58106. Zbl 764.58026.

[4] C. Bernhardt and E. M. Coven, A polynomial-time algorithm for deciding the forcing relation on cyclic permutations, Symbolic Dynamics and its Applications (New Haven, CT, 
1991) (Rhode Island), Contemp. Math., vol. 135, Amer. Math. Soc., 1992, pp. 85-93. MR 93j:58041. Zbl 770.58016.

[5] P. Collet and J.-P. Eckmann, Iterated Maps on the Interval as Dynamical Systems, Progress in Physics, vol. 1, Birkhäuser, Massachusetts, 1980. MR 82j:58078. Zbl 458.58002.

[6] I. Jungreis, Some results on the Šarkovskil partial ordering of permutations, Trans. Amer. Math. Soc. 325 (1991), no. 1, 319-344. MR 91h:58060. Zbl 715.58028.

[7] I. Mulvey, Symbolic representation for a class of unimodal cycles, Topology and its Applications, to appear in Int. J. Math. Math. Sci.

[8] A A geometric algorithm to decide the forcing relation on cycles, Real Anal. Exchange 23 (1997/98), no. 2, 709-717. MR 99j:58164. Zbl 939.37021.

[9] _ On the structure of the totally ordered set of unimodal cycles, Int. J. Math. Math. Sci. 25 (2001), no. 5, 323-329.

IRENE Mulvey: DePARTMENT OF MATHEMATICS AND COMPUTER SCIENCE, FAIRFIELD UNIVERSITY, FAIRFIELD, CT 06430, USA

E-mail address: mu1vey@fair1. fairfie1d.edu 


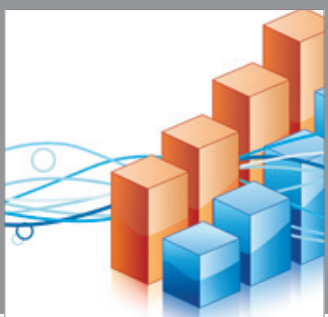

Advances in

Operations Research

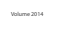

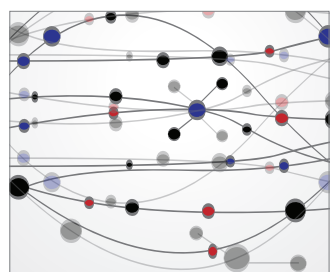

\section{The Scientific} World Journal
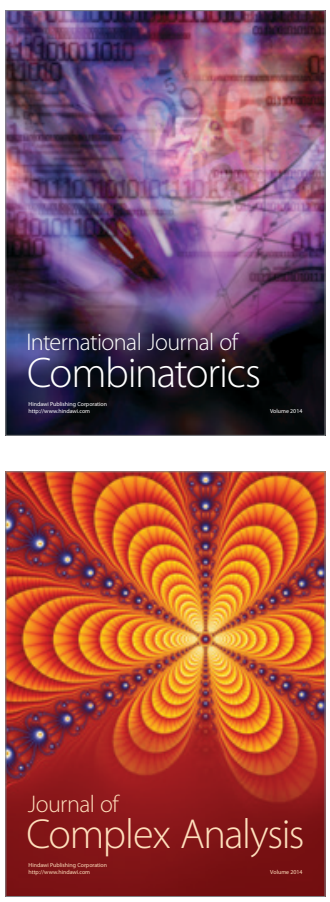

International Journal of

Mathematics and

Mathematical

Sciences
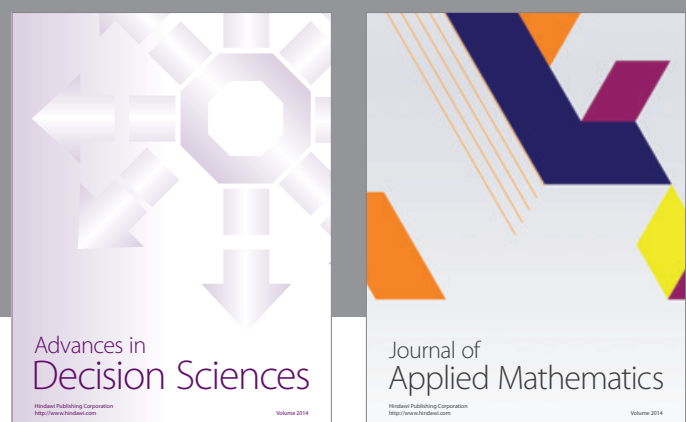

Journal of

Applied Mathematics
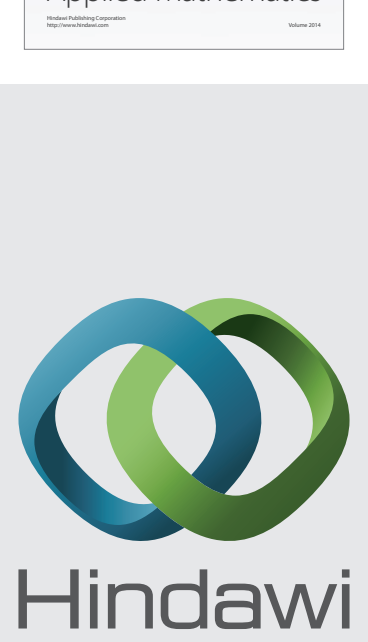

Submit your manuscripts at http://www.hindawi.com
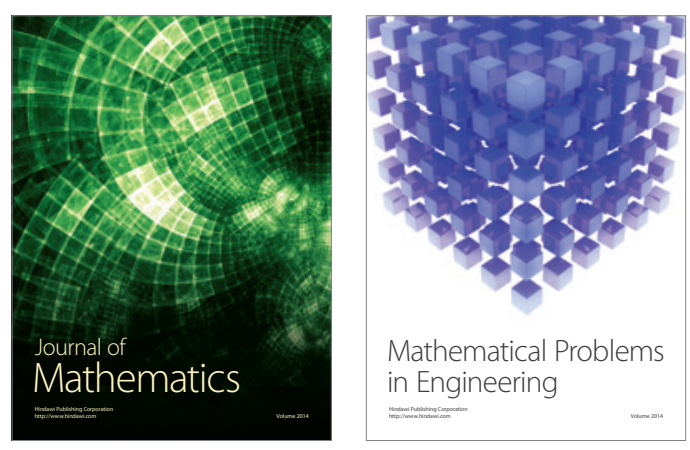

Mathematical Problems in Engineering
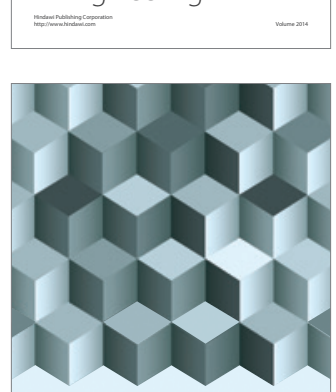

Journal of

Function Spaces
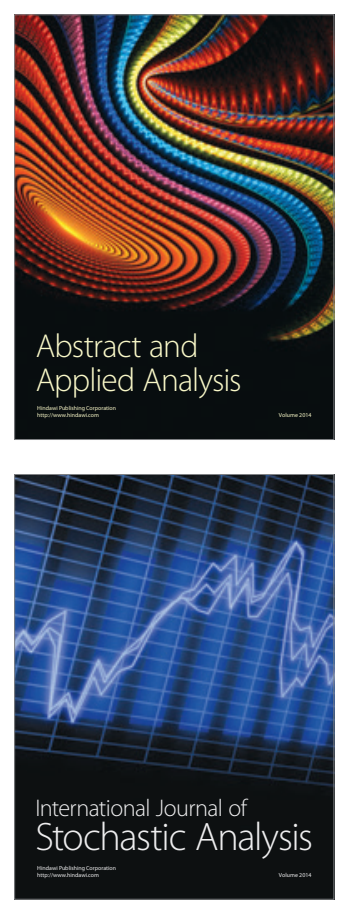

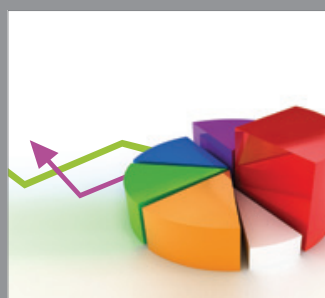

ournal of

Probability and Statistics

Promensencen
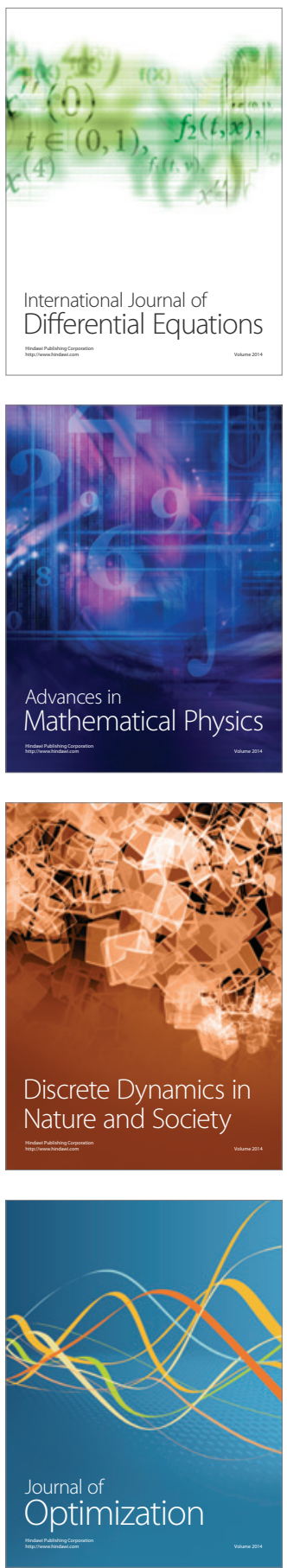